\section{A case of early gastric cancer accompanied by a hamartomatous inverted polyp and successfully managed with endoscopic submucosal dissection}
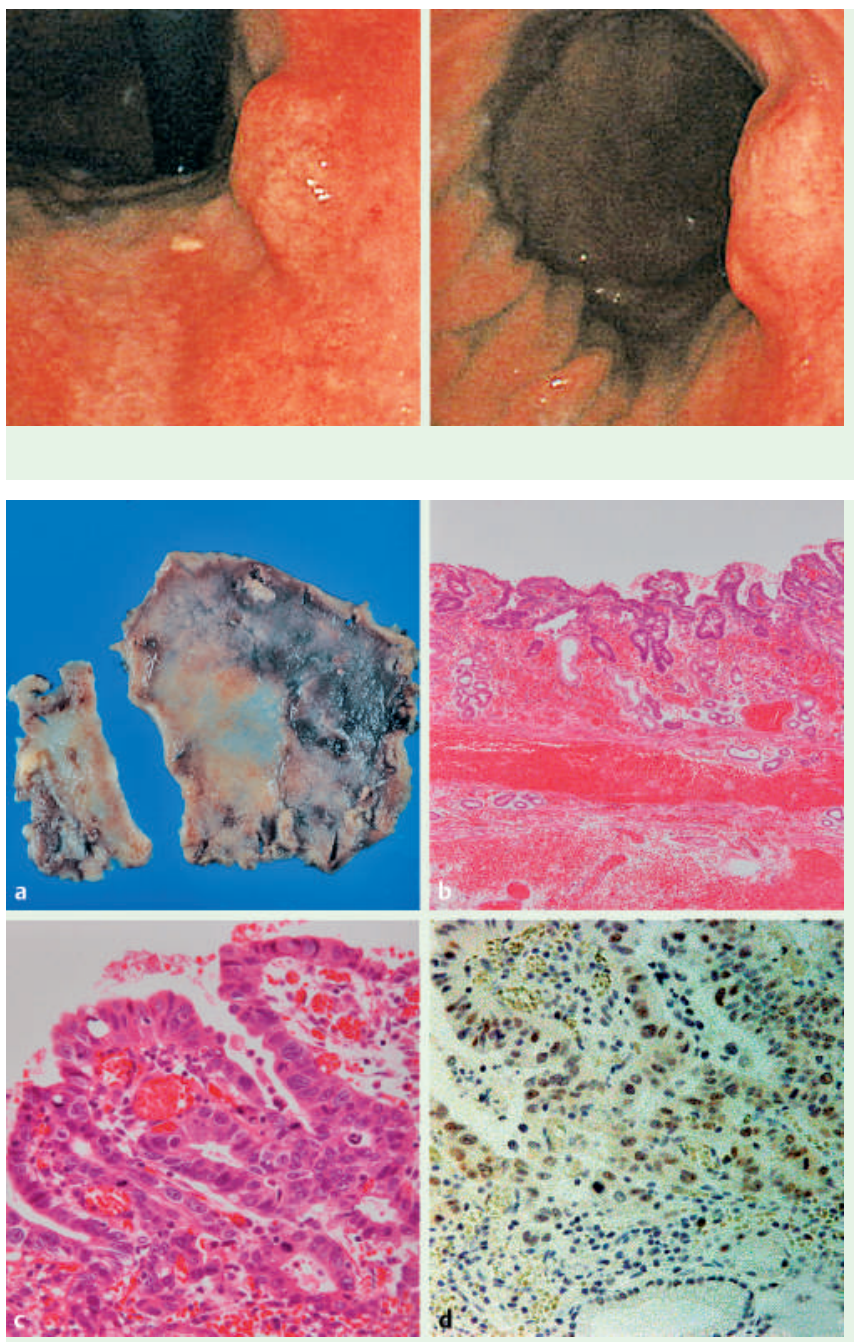

Figure 1 The upper gastrointestinal endoscopy revealed a submucosal tumor (SMT) in the posterior wall of the upper third area of the stomach, with bridging fold and positive cushion sign. The biopsy of discolored mucosa at the top of the SMT indicated the possibility of adenocarcinoma.

Figure 2 After circumferential incision of mucosa all around the submucosal tumor (SMT), the resected piece was separated as far as possible. We could not lift it with injection agent, especially just under the SMT. We therefore removed the mucosa, including the SMT, in two blocks with snare resection (a). Pathologic examinations revealed irregular tubular structures limited to the mucosal layer (b, c), with p53positive immunohistochemical staining (d). In the submucosal layer, cystic dilated glands without atypia were observed (b) and were p53 negative (d).

A submucosal tumor (SMT) was found during a regular health check-up in a 59year-old man. Following barium meal study the SMT was shown to be growing. He visited our hospital for further examination.

The upper gastrointestinal endoscopy revealed an SMT in the posterior wall of the upper third area of the stomach, with bridging fold and positive cushion sign (৫ Figure 1). A focal discoloration of mucosa at the top of the SMT indicated the possibility of co-existence of gastric cancer. We therefore performed a biopsy of this mucosa, and irregular tubular structures were observed on pathologic studagnostic therapy. surface of the HIP. without atypia, accompanied by dendritic proliferation of the smooth muscle bundle [1]. HIP is very rare and difficult to diagnose. In addition, HIP is reported as a paracancerous lesion, and is associated with gastric cancers $[1,2]$. Therefore, we have to diagnose it immediately and carefully with these points in mind during endoscopic studies, including biopsy. However, a superficial biopsy cannot be effective for critical diagnosis of submucosal lesions.

We propose that aggressive biopsy of SMT is necessary if equivocal findings are noted. Endoscopic submucosal dissection can be an effective procedure of diagnostic therapy for HIP and early cancer. Thus, HIP can be one of the differential diagnoses of SMT lesions of the stomach, and can be occasionally associated with early gastric cancer.

Endoscopy_UCTN_Code_CCL_1AB_2AD_3AB

S. Ono ${ }^{1}$, T. Kamoshida ${ }^{1}$, Y. Hiroshima ${ }^{1}$,

A. Okawara ${ }^{1}$, T. Matsuo ${ }^{1}$, N. Kakinoki ${ }^{1}$,

A. Ishikawa ${ }^{1}$, Y. Kishimoto ${ }^{1}$, S. Hirai ${ }^{1}$, Y. Oka ${ }^{1}$, T.Shimokama ${ }^{2}$

1 Department of Internal Medicine, Hitachi General Hospital, Hitachi, Japan

2 Department of Pathology, Hitachi

General Hospital, Hitachi, Japan

\section{References}

1 Mandai K, Moriwaki S, Doihara H et al. Diffuse heterotopic glandular cysts of the stomach as a paracancerous lesion of gastric carcinoma (in Japanese with English abstract). Byori To Rinsho 1991; 9: 1217 1225

2 Iwanaga T, Koyama H, Furukawa H et al. Diffuse submucosal cysts in the stomach as probable paracancerous lesion (in Japanese with English abstract). Nippon Shokakibyo Gakkai Zasshi 1976; 73: 31 - 40

Bibliography

DOI 10.1055/s-2007-966482

Endoscopy 2007; 39: E202

(c) Georg Thieme Verlag KG Stuttgart · New York . ISSN 0013-726X mucosa around the SMT. Endoscopic submucosal dissection was carried out for di-

Pathologic examinations revealed irregular tubular structures limited to the mucosal layer, and an immunohistochemical test revealed p53-positive staining ( $\bullet$ Figure 2). In the submucosal layer, cystic dilated glands without atypia were observed, compatible with hamartomatous inverted polyp (HIP). These facts indicated early gastric cancer (IIb) on the

HIP is characterized pathologically as a proliferation of cystic dilated glands
Corresponding author

\section{S. Ono, MD}

Department of Internal Medicine

Hitachi General Hospital

2-1-1 Jonan-cho

Hitachi

Ibaraki

Japan

Fax: +81-294-238351

satoshi-tky@umin.ac.jp 